\title{
Enraizamiento de estacas de Pinus hartwegii de tres poblaciones naturales en ecosistemas de alta montaña del Estado de México y Veracruz
}

\author{
Rooting of Pinus hartwegii cuttings from three natural populations \\ in high-mountain ecosystems from Mexico and Veracruz State
}

\author{
Francisco Rivera Melo a, Marcos Jiménez Casas a*, \\ Carlos Ramírez Herrera a , Alma Yadira Martínez Rendón b
}

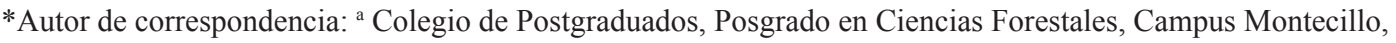
Carretera México-Texcoco, km 36,5, Montecillo, México, código postal 56230, tel.: (595) 9520200 ext. 1454, marcosjc@colpos.mx

${ }^{\mathrm{b}}$ Universidad Nacional Autónoma de México, Instituto de Biología,

Laboratorio Regional de Biodiversidad y Cultivo de Tejidos Vegetales, Santa Cruz Tlaxcala, Tlaxcala, México.
\end{abstract}

\begin{abstract}
SUMMARY
The limited seed production of Pinus hartwegii Lidl. requires the use of vegetative propagation techniques to produce plants for reforestation of the high mountain ecosystems. Nothing about vegetative propagation of the species has been done to our knowledge, therefore its ability to root propagules is, so far, unknown. In this study, the rooting of cuttings from three populations of $P$. hartwegii was evaluated, testing three treatments of Indole Butyric Acid (IBA, Radix $\left.{ }^{\circledR}\right)(0,5,000$ and 10,000 ppm). The experiment was established in rooting chambers under a randomized four-block design with factorial arrangement. The rooted cuttings were transplanted and kept in a greenhouse. Survival, number of rooted cuttings, length and number of primary and secondary roots were evaluated after 12 weeks. Regardless of the auxin treatment, high percentages of rooting were observed in the cuttings. The auxin in 5,000 ppm amplified and improved the response to rooting $(96.6 \%)$; while the concentration of 10,000 ppm caused toxicity, which was reflected in the lower survival of the cuttings $(63.5 \%)$. The cuttings of the three populations showed high percentages of survival (> $80 \%)$ and rooting (>67\%); although without significant differences among them. A hundred percent of the rooted cuttings survived transplant. Massive multiplication through cuttings rooting is feasible for $P$. hartwegii, regardless of the genotype. With the application of exogenous auxins $(5,000 \mathrm{ppm})$ the regeneration and development of roots was favored.
\end{abstract}

Key words: Indole Butyric Acid, vegetative propagation, adventitious roots, high mountain pines.

\section{RESUMEN}

La limitada producción de semilla de Pinus hartwegii Lidl. requiere del uso de técnicas de propagación vegetativa para producir plantas demandadas para la reforestación de los ecosistemas de alta montaña. Nada sobre propagación vegetativa de la especie se ha hecho, por lo que su capacidad para enraizar propágulos es desconocida. En este estudio se evaluó el enraizamiento de estacas de tres poblaciones de $P$. hartwegii (Nevado de Toluca, Monte Tláloc y Cofre de Perote), probando tres tratamientos de ácido indolbutírico (AIB, Radix ${ }^{\circledR}$ ) $(0,5.000$ y $10.000 \mathrm{ppm})$. Estas se establecieron en cámaras de enraizamiento bajo un diseño de bloques al azar con arreglo factorial. Las estacas enraizadas fueron trasplantadas y mantenidas en invernadero. La supervivencia, el número de estacas enraizadas, longitud y número de raíces primarias y secundarias se evaluaron después de 12 semanas. Independientemente del tratamiento de auxinas, se registraron altos porcentajes de estacas enraizadas. Con la auxina en concentración de 5.000 ppm se amplificó y mejoró la respuesta al enraizamiento (93,3\%); mientras que la concentración de 10.000 ppm causó toxicidad, reflejada en el decremento de supervivencia de las estacas $(64,6 \%)$. Las estacas de las tres poblaciones mostraron altos porcentajes de supervivencia (> $80 \%)$ y enraizamiento ( $>67 \%)$; aunque sin diferencias significativas entre ellas. El $100 \%$ de las estacas enraizadas sobrevivieron al trasplante. Se demostró que la multiplicación masiva a través del enraizamiento de estacas es factible para $P$. harwegii, independientemente del genotipo, con la aplicación de auxinas exógenas (5.000 ppm) se favoreció la regeneración y desarrollo de raíces.

Palabras clave: ácido indolbutírico, propagación vegetativa, raíces adventicias, pinos de alta montaña.

\section{INTRODUCCIÓN}

Pinus hartwegii Lindl. es una especie típica de las zonas templadas, confinada a los límites altitudinales $(2.800$ a 4.300 m s.n.m.) de la vegetación arbórea en México y, muy importante, en los servicios ecosistémicos de regulación (Alfaro-Ramírez et al. 2020). La especie tiene ciclos semilleros hasta de cinco años y, en las últimas décadas, 
su producción de semilla ha decrecido dramáticamente, lo cual se refleja en una limitada regeneración natural (Tejeda-Landero et al. 2019). Algunos estudios señalan que la especie tendrá una disminución en su área de distribución de hasta el $80 \%$, con impacto negativo para su ecosistema (Gutiérrez y Trejo 2014). Por lo tanto, es necesario generar sistemas de producción de planta, en el corto plazo, para apoyar a los programas de reforestación y contribuir en su regeneración artificial.

El enraizamiento de estacas es un método de propagación que ha dado buenos resultados en las especies de pino, pero es importante contar con buena producción de estacas juveniles en la planta madre y adecuar el protocolo según la especie en cuestión (Majada et al. 2011, Matsunaga y Ohira 2019, Nugrahanto et al. 2019, Escamilla-Hernández et al. 2020). La aplicación de este sistema ha sido relevante en el sector forestal ya que permite multiplicar genotipos superiores con características deseables, aprovechar las ganancias genéticas obtenidas durante los ciclos de mejoramiento con la clonación, incrementar y uniformizar el rendimiento de las plantaciones forestales comerciales y conservar genotipos en peligro de extinción (White et al. 2007).

A pesar de su estado cespitoso, las plántulas de $P$. hartwegii desarrollan naturalmente ramillas basales con características juveniles y que pueden aprovecharse como estacas. Además, hemos observado, en trabajos de manejo de la planta, que estas ramillas también pueden ser inducidas en la plántula a través de podas periódicas programadas. Sin embrago, la capacidad de enraizamiento de estas ramillas es desconocida. En general, las especies del género Pinus son consideradas de difícil enraizamiento (Bonga et al. 2010).

Es deseable propagar genotipos de varias poblaciones de P. hartwegii y contar con germoplasma de cada localidad, ya que la variación genética entre poblaciones se debe considerar para evitar el movimiento geográfico de genotipos y afectar el éxito de la reforestación (Flores-García et al. 2020). Sin embargo, la respuesta al enraizamiento puede ser variable entre los propágulos de cada población, desde que se ha señalado que la inducción de las raíces adventicias depende en gran medida del genotipo, aun siendo de la misma especie (Hakamata et al. 2016). Factores fisiológicos, genéticos y ambientales han sido estudiados en especies difíciles de enraizar, concluyendo que las auxinas exógenas promueven e incrementan la capacidad de enraizamiento de las estacas, pero la concentración adecuada de auxina varía en función del genotipo o especie (Ragonezi et al. 2010).

El ácido indolbutírico (AIB) es la auxina sintética más usada para inducir el enraizamiento de estacas, ya que su efecto promotor es consistente, particularmente en especies forestales maderables (Pacurar et al. 2014). En pinos mexicanos, la efectividad del AIB en el enraizamiento de estacas fue comprobada en investigaciones realizadas con Pinus leiophylla (Schiede ex Schltdl. et Cham.) y Pinus patula (Schiede ex Schltdl. et Cham.), pero la concentración óptima de AIB dependió de la especie (Cuevas-Cruz et al. 2015; Bautista-Ojeda 2020).
En $P$. hartwegii no existe información sobre propagación vegetativa. Por lo que es importante investigar la capacidad de enraizamiento que tienen sus estacas, para proponer nuevas estrategias de producción de plantas. Lo cual apoyará a los programas de reforestación y conservación del ecosistema de esta especie. El objetivo de este trabajo es evaluar el efecto del ácido indolbutírico (AIB) en el enraizamiento de estacas procedentes de tres poblaciones (Cofre de Perote, Monte Tláloc y Nevado de Toluca) de $P$. hartwegii. Como hipótesis se planteó que el AIB incrementará el enraizamiento diferencialmente en las estacas de las tres poblaciones consideradas, puesto que esta auxina ha sido la más efectiva para enraizar estacas en las especies forestales maderables hasta ahora probadas, particularmente en especies de Pinus, pero su efectividad estará en función del genotipo.

\section{MÉTODOS}

El trabajo se realizó en el invernadero (cubierta de polietileno, $90 \%$ de luz, $45-80 \% \mathrm{HR}$ y $18-32{ }^{\circ} \mathrm{C}$ ) del Posgrado en Ciencias Forestales del Colegio de Postgraduados, Campus Montecillo, ubicado entre las coordenadas geográficas $19^{\circ} 27^{\prime} 34.8^{\prime \prime} \mathrm{N}$ y $98^{\circ} 54^{\prime} 15.8^{\prime \prime} \mathrm{O}$, a una altitud de 2.249 m s.n.m., en el Municipio de Texcoco, Estado de México. Las semillas utilizadas en este experimento para la producción de las plantas madres fueron recolectadas en tres poblaciones naturales de $P$. hartwegii: 1) Monte Tláloc, Estado de México; 2) Nevado de Toluca, Estado de México y 3) Cofre de Perote, Veracruz (cuadro 1).

La recolección se realizó entre noviembre y febrero de los años 2017 y 2018, en árboles libres de plagas y enfermedades, pero sin considerar la distancia entre árboles, debido a los pocos individuos con presencia de conos en los rodales muestreados. Los conos fueron trasladados al laboratorio para la extracción y separación de las semillas llenas y vacías.

Producción de plantas madres. En febrero del 2019, previo a su siembra, las semillas fueron sometidas a un tratamiento

Cuadro 1. Ubicación geográfica de las tres poblaciones de Pinus hartwegii consideradas en el estudio.

Geographical location of the three Pinus hartwegii populations considered in the study.

\begin{tabular}{|c|c|c|}
\hline Población & Altitud (m s.n.m.) & Coordenadas \\
\hline Monte Tláloc & 3.700 & $\begin{array}{l}19^{\circ} 24^{\prime} 52,11^{\prime \prime} \mathrm{N} \\
98^{\circ} 44^{\prime} 29,66^{\prime \prime} \mathrm{O}\end{array}$ \\
\hline Nevado de Toluca & 4.000 & $\begin{array}{l}19^{\circ} 07^{\prime} 25,66^{\prime \prime} \mathrm{N} \\
99^{\circ} 46^{\prime} 43,77^{\prime \prime} \mathrm{O}\end{array}$ \\
\hline Cofre de Perote & 3.800 & $\begin{array}{l}19^{\circ} 30^{\prime} 19,42^{\prime \prime} \mathrm{N} \\
97^{\circ} 09^{\prime} 31,37^{\prime \prime} \mathrm{O}\end{array}$ \\
\hline
\end{tabular}


pre-germinativo y de desinfección, con el propósito de estimular y homogeneizar la germinación, así como eliminar posibles patógenos potenciales. En este tratamiento, las semillas se sumergieron en una solución elaborada con agua oxigenada al $0,3 \%$ e hipoclorito de sodio comercial (Cloralex ${ }^{\circledR}$, Comercializadora Chrisalim S.A de C.V) al $2 \%$, durante un periodo de 24 horas.

Producción y manejo de plantas madre. La siembra se realizó en contenedores de $310 \mathrm{ml}$, colocando una semilla por tubete forestal en sustrato, previamente esterilizado en cámara de vapor, conformado por una mezcla de turba, agrolita y vermiculita $(3: 1: 1 \mathrm{v} / \mathrm{v})$, al cual se agregó un fertilizante de liberación controlada de 4 meses, Multicote ${ }^{\mathrm{TM}}$ $\left(15-7-15+2 \mathrm{MgO}+\right.$ micronutrientes), a una dosis de $4 \mathrm{~g} \mathrm{~L}^{-1}$ de sustrato. Semanalmente se realizaron aplicaciones, por aspersión, al sustrato de fungicida (Captan ${ }^{\circledR} 2 \mathrm{~g} \mathrm{~L}^{-1}, \mathrm{~N}$ (triclorometiltio) ciclohex-4-eno-1,2-dicarboximida) para evitar la contaminación de la planta madre. Las plantas se mantuvieron en condiciones de invernadero (60-80\% HR y $18-30{ }^{\circ} \mathrm{C}$ ), con riegos por aspersión proporcionados tres veces por semana; y fertilizadas con el fertilizante comercial Peters $^{\mathrm{TM}}$ (macro y micro nutrimentos a base de nitrógeno, fósforo y potasio en proporciones de 10-30-20, respectivamente), aplicado cada 15 días, en una concentración de $1 \mathrm{~g} \mathrm{~L}^{-1} \mathrm{y}$ ajustando a $\mathrm{pH}$ de 5,5.

En mayo del 2019, las plantas se trasplantaron a contenedores de $1 \mathrm{~L}$ para permitir un mejor desarrollo y crecimiento de la raíz y de la planta en general, bajo las condiciones de invernadero previamente señaladas. La frecuencia de riego y fertilización fue la misma, pero para este caso se usó la proporción 20-20-20 del mismo fertilizante, recomendada para la fase de desarrollo de la planta, con una concentración de $1,5 \mathrm{~g} \mathrm{~L}^{-1} \mathrm{y} \mathrm{pH}$ de 5,5. Para el experimento 40 plantas madre por población fueron seleccionadas por el tamaño y número de ramas basales.

Establecimiento del ensayo. El experimento de enraizado de estacas se inició el 15 de octubre de 2019 cuando las plantas madre o donadoras de estacas cumplieron los nueve meses de edad y presentaban un promedio de seis ramas basales con longitud mayor a $5 \mathrm{~cm}$. Las ramas basales consideradas como esquejes, se recolectaron con un bisturí, evitando dañar el eje principal de la planta. La zona de corte de la planta madre fue tratada mediante pulverización con solución fungicida (Captan ${ }^{\circledR} 1 \mathrm{~g} \mathrm{~L}^{-1}$ ), con el fin de evitar contaminaciones. Una vez obtenidas, las estacas se sumergieron en la misma solución fungicida y permanecieron en agitación manual constante durante cinco minutos. Finalmente, las estacas fueron extraídas de la solución y se les realizó un corte de forma diagonal en la base, donde se aplicó 3 concentraciones de la auxina, ácido indolbutírico Radix ${ }^{\circledR}$ (producto comercial en polvo, Ácido Indol-3-Butírico (4-(1 H-Indol-3-yl)-1.0\%, Ingredientes Inertes-99.0 \%. Intercontinental Import Export). Para el caso del tratamiento testigo $(0 \mathrm{ppm})$ se usó talco industrial inerte. Cada estaca tuvo una longitud de entre 5 y $8 \mathrm{~cm}$, teniendo área disponible para ser enterrada en el sustrato, a profundidad de $3 \mathrm{~cm}$ desde la base de la estaca.

El sustrato estéril empleado para el enraizamiento fue diferente en cuanto a su proporción al señalado anteriormente y consistió en una mezcla de agrolita, vermiculita y turba en relación 3:1:1 v/v, respectivamente. El sustrato fue colocado en una cámara de enraizado cubierta con polietileno previamente tratada con fungicida. El sustrato fue regado por aspersión hasta saturación. La humedad relativa de la cámara se mantuvo en el intervalo 80-90 \% mediante aspersión manual, y monitoreada mediante el sensor data logger Temp/Hum HOBO ${ }^{\circledR}$ (Model U10-003).

Posteriormente al establecimiento de las estacas se aplicó fungicida (Captan ${ }^{\circledR} 1 \mathrm{~g} \mathrm{~L}^{-1}$ ) e insecticida (Engeo ${ }^{\circledR}$ $1 \mathrm{ml} \mathrm{L}^{-1}$, Thiamethoxam $141 \mathrm{~g} / \mathrm{L}$, Lambda-cyhalothrin 106 $\mathrm{g} / \mathrm{L}$ ) cada 8 días para evitar contaminación o ataque de plagas en los esquejes.

Diseño experimental. Para este estudio se utilizó un diseño experimental de bloques completos al azar con arreglo factorial $3 \times 3$; el factor auxina con tres niveles ( 0 testigo, 5.000 y $10.000 \mathrm{ppm}$ ) y el factor población con tres niveles (Cofre de Perote, Monte Tláloc y Nevado de Toluca), el experimento fue replicado cuatro veces y se tuvieron ocho estacas por unidad experimental, por lo que se usaron un total de 288 estacas.

La evaluación se realizó a las 12 semanas después de haber establecido el experimento (figura $1 \mathrm{~A}$ ), las variables respuesta evaluadas fueron: estacas vivas (\%), estacas enraizadas (\%), número y longitud de raíces primarias y número de raíces secundarias (figura $1 \mathrm{~B}$ ). Se consideró como una estaca enraizada cuando había presencia de raíz mayor o igual a $1 \mathrm{~mm}$ de longitud; mientras que la estaca muerta se consideró cuando había necrosis en más del 60 \% del tejido.

Generalmente las estacas producen más de tres raíces adventicias (figura $1 \mathrm{~B}$ ), pero ninguna domina en tamaño para ser considerada como raíz principal. Por lo que, en este trabajo, a estas raíces se les denominó raíces primarias. La longitud de la raíz primaria se obtuvo con el promedio de la longitud de todas las raíces primarias presentes en la estaca. Después de evaluar el sistema radical formado las estacas enraizadas fueron trasplantadas a tubete forestal (capacidad $310 \mathrm{~cm}^{3}$ ) con la misma mezcla de sustrato usada en la producción de la planta madre (figura $1 \mathrm{C}$ y D) y el porcentaje de supervivencia tras el trasplante se registró después de tres y seis meses. Las plantas fueron mantenidas en invernadero.

Análisis estadístico. En el análisis estadístico se utilizaron los valores promedio por unidad experimental de la supervivencia y enraizamiento (\%), número raíces primarias, secundarías y longitud de raíces primarias, a través de un modelo lineal para el diseño factorial considerado [1]. Debido a que los datos en la escala original no cumplían con 


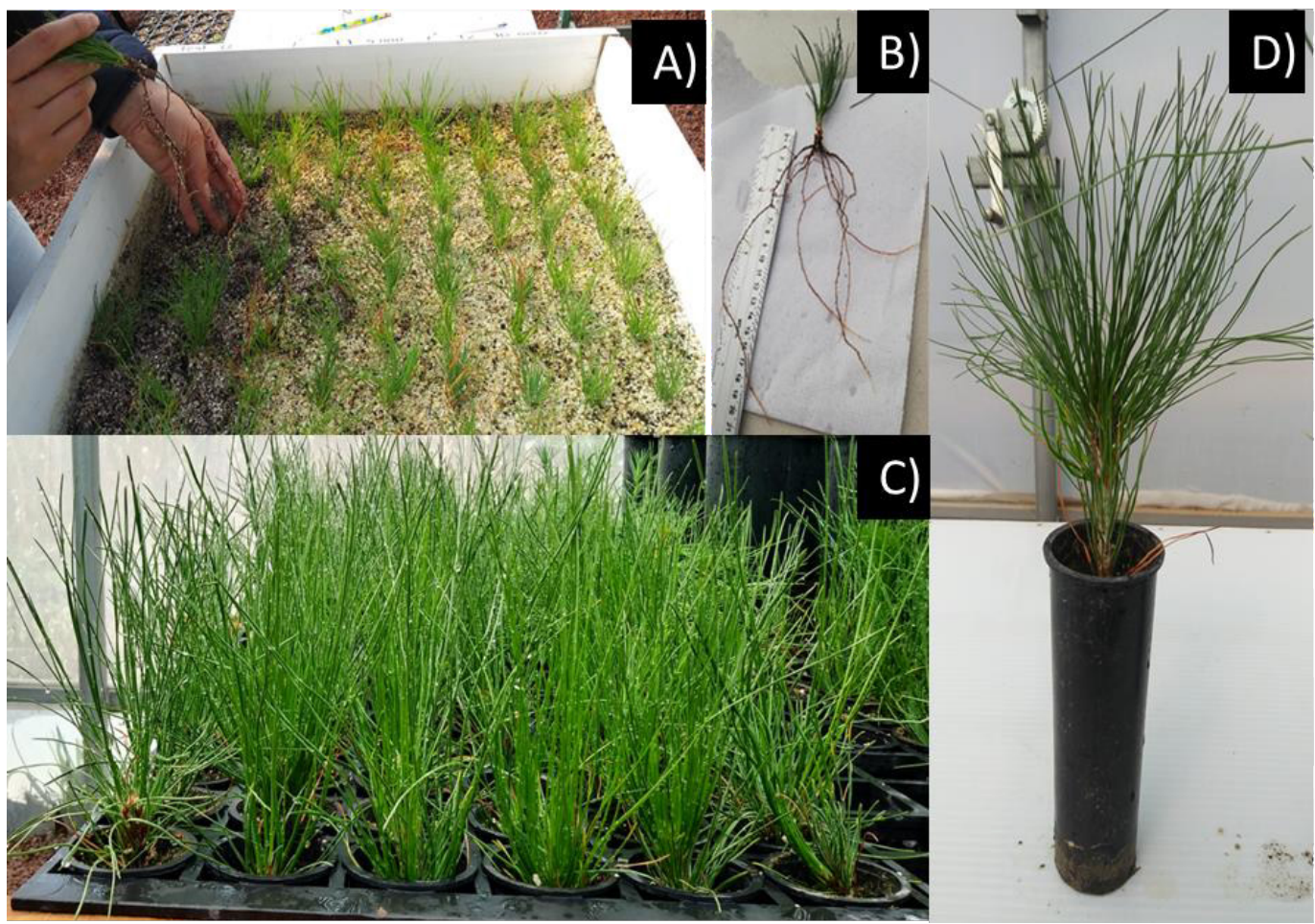

Figura 1. Esquema general del experimento establecido para la producción de planta de Pinus hartwegii por enraizamiento de estacas, probando concentraciones de AIB en estacas de tres poblaciones. A) Evaluación de estacas después de 3 meses en cámara de enraizamiento; B) Estaca enraizada con varias raíces adventicias consideradas como principales en este experimento; C) Estacas enraizadas después de tres meses de trasplante a tubete forestal y D) Planta de seis meses de edad, producida por estaca enraizada.

General scheme of the experiment established for the production of Pinus hartwegii plants by rooting cuttings, testing IBA concentrations in cuttings from three populations. A) Cuttings harvest after 3 months in rooting chamber; B) Rooted cutting with several adventitious roots considered as main root in this experiment; C) Rooted cuttings after three months of transplanting to forest tubes and D) Six-month-old seedling, produced by rooted cuttings.

los criterios de normalidad y homogeneidad de varianza, se utilizó el procedimiento GLIMMIX del paquete estadístico SAS, versión 9.3. Procedimiento que permite trabajar con datos que no muestran una distribución normal. En las variables donde se encontró diferencia significativa se realizó la prueba de comparación de medias con Tukey $(P \leq 0,05)$.

$$
y_{i j k}=\mu+\beta_{k}+A_{i}+P_{j}+A P_{i j}+\varepsilon_{i j k}
$$

Donde, $\mu=$ media general, $\beta k=$ efecto del bloque, $A i=$ efecto fijo de concentración de $i$ en el factor auxina (tres niveles), $P_{j}=$ efecto aleatorio del nivel de $j$ en el factor población (tres niveles), $A P i j=$ interacción $A^{*} P$, correspondiente al nivel $i$ de $A$ y nivel $j$ de $P$, y $\varepsilon$ ijk $=$ error experimental.

\section{RESULTADOS}

El análisis de varianza mostró efecto significativo del AIB $(P<0,05)$ para todas las variables consideradas, excepto, para la longitud de raíces primarias; mientras que el factor población únicamente mostró diferencias para el número de raíces primarias y secundarias. Por otro lado, la interacción entre los dos factores probados solo fue significativa para el número de raíces secundarias (cuadro 2).

Efecto de la concentración de auxina. El tratamiento con la concentración de 10.000 ppm de AIB causó un decremento en la supervivencia de las estacas de aproximadamente $35 \%$, con respecto a las estacas tratadas con concentraciones de 5.000 y 0 ppm de AIB; las cuales mostraron porcentajes de supervivencia similares, con solo una mortalidad menor al $5 \%$ (cuadro 3 ).

Respecto a la inducción y producción de raíces adventicias, las estacas sometidas al tratamiento $5.000 \mathrm{ppm}$ de AIB tuvieron la mejor respuesta a la generación de raíces, ya que incrementaron significativamente el porcentaje de estacas con raíz en más del $47 \%$, respecto al tratamiento testigo; y aunque sin significancia estadística en $8 \%$ en relación con las tratadas con 10.000 ppm de AIB (cuadro 3).

Por otro lado, la presencia de AIB en las estacas promovió de manera importante la formación de raíces de primer y segundo orden. Las de primer orden tuvieron la mayor producción con 10.000 ppm de AIB, incrementando en 25 
y $70 \%$ la producción, en relación con las del tratamiento de 5.000 y 0 de AIB, respectivamente. La producción de las de segundo orden fue similar entre los tratamientos de auxina, los cuales registraron $46 \%$ más raíces que las estacas testigo (cuadro 3).

En el promedio de la longitud de la raíz principal no fue afectada por los tratamientos; aunque ligera reducción, no significativa, de aproximadamente $14 \%$ se observó en las estacas tratadas con $10.000 \mathrm{ppm}$ de AIB, respecto a las de los otros dos tratamientos (cuadro 3). En general, las raíces presentaron un buen vigor, forma y tamaño (mayor de $7 \mathrm{~cm}$ ) requeridos para su trasplante a contenedor.

Efecto de la población. La supervivencia, enraizamiento y longitud de la raíz principal de las estacas no tuvieron variación estadísticamente significativa entre las tres poblaciones; a diferencia de la producción de raíces de primer y segundo orden (cuadro 4). Entre las poblaciones la

Cuadro 2. Valores de significancia (P) obtenidos en el análisis de varianza para supervivencia de estacas (\%), enraizamiento de estacas (\%), número de raíces, y longitud de raíces las variables registradas en el experimento de enraizamiento de estacas de las tres poblaciones (Tláloc, Cofre de perote y Nevado de Toluca) de Pinus hartwegii.

Significance values (p) obtained in the analysis of variance for the variables recorded in the rooting experiment of cuttings of three populations of Pinus hartwegii.

\begin{tabular}{|c|c|c|c|c|c|}
\hline \multirow{2}{*}{ Fuente de variación } & \multirow{2}{*}{ Supervivencia (\%) } & \multirow{2}{*}{ Enraizamiento (\%) } & \multicolumn{2}{|c|}{ Número de raíces } & \multirow[t]{2}{*}{ Longitud de raíces primarias } \\
\hline & & & Primarias & Secundarias & \\
\hline Ácido indolbutírico (AIB) & $<0,0001^{*}$ & $<0,0001 *$ & $<0,0001 *$ & $0,0005^{*}$ & 0,0950 \\
\hline Población & 0,2625 & 0,1333 & $0,0004 *$ & $0,0239^{*}$ & 0,7822 \\
\hline AIB*Población & 0,1166 & 0,9841 & 0,3761 & $0,0059 *$ & 0,3876 \\
\hline
\end{tabular}

* Con diferencias significativas $(P \leq 0,05)$.

Cuadro 3. Valores medios ( \pm error estándar) por dosis de AIB $(0,5.000$ y 10.000) en la supervivencia de estacas (\%), estacas enraizadas (\%), número de raíces y longitud de raíces primarias evaluadas en el experimento de enraizamiento de estacas de Pinus hartwegii.

Mean values ( \pm standard error) per IBA dose in the variables evaluated in the rooting experiment of Pinus hartwegii cuttings.

\begin{tabular}{|c|c|c|c|c|c|}
\hline \multirow{2}{*}{$\mathrm{AIB}(\mathrm{ppm})$} & \multirow{2}{*}{ Supervivencia (\%) } & \multirow{2}{*}{ Estacas enraizadas (\%) } & \multicolumn{2}{|c|}{ Número de raíces } & \multirow{2}{*}{ Longitud de raíces primarias $(\mathrm{cm})$} \\
\hline & & & Primarias & Secundarias & \\
\hline 0 & $98,9 \pm 1,0 \mathrm{a}$ & $49,4 \pm 3,81 \mathrm{~b}$ & $2,7 \pm 0,4 \mathrm{c}$ & $19,5 \pm 2,9 \mathrm{~b}$ & $9,4 \pm 0,7 \mathrm{a}$ \\
\hline 5.000 & $96,9 \pm 2,2 \mathrm{a}$ & $93,3 \pm 2,99 \mathrm{a}$ & $7,3 \pm 0,8 b$ & $36,7 \pm 4,7 \mathrm{a}$ & $9,2 \pm 0,4 \mathrm{a}$ \\
\hline 10.000 & $64,6 \pm 6,5 b$ & $85,3 \pm 6,7 \mathrm{a}$ & $9,8 \pm 0,6 \mathrm{a}$ & $31,7 \pm 2,1 \mathrm{a}$ & $7,1 \pm 0,4 \mathrm{a}$ \\
\hline
\end{tabular}

* Letras diferentes indican diferencias significativas $(P \leq 0,05)$.

Cuadro 4. Valores medios ( \pm error estándar) de acuerdo con la población en las variables supervivencia de estacas (\%), estacas enraizadas (\%), número de raíces y longitud de raíces primarias evaluadas en el experimento de enraizamiento de estacas de Pinus hartwegii.

Mean values ( \pm standard error) according to the population in the variables evaluated in the rooting experiment of Pinus hartwegii cuttings.

\begin{tabular}{|c|c|c|c|c|c|}
\hline \multirow{2}{*}{ Población } & \multirow{2}{*}{ Supervivencia (\%) } & \multirow{2}{*}{ Estacas enraizadas (\%) } & \multicolumn{2}{|c|}{ Número de raíces } & \multirow{2}{*}{ Longitud de raíces primarias $(\mathrm{cm})$} \\
\hline & & & Primarias & Secundarias & \\
\hline Nevado de Toluca & $91,7 \pm 3,8 \mathrm{a}$ & $80,3 \pm 6,2 \mathrm{a}$ & $6,0 \pm 0,8 \mathrm{~b}$ & $32,8 \pm 4,2 \mathrm{a}$ & $8,3 \pm 0,6 \mathrm{a}$ \\
\hline Monte Tláloc & $83,3 \pm 6,9 \mathrm{a}$ & $80,7 \pm 6,0 \mathrm{a}$ & $5,3 \pm 0,9 \mathrm{~b}$ & $32,3 \pm 4,1 \mathrm{ab}$ & $8,9 \pm 0,8 \mathrm{a}$ \\
\hline Cofre de Perote & $85,4 \pm 7,0 \mathrm{a}$ & $66,9 \pm 9,0 \mathrm{a}$ & $8,5 \pm 1,3 \mathrm{a}$ & $22,8 \pm 2,9 b$ & $8,5 \pm 0,6 \mathrm{a}$ \\
\hline
\end{tabular}

Letras diferentes indican diferencias significativas $(P \leq 0,05)$. 
supervivencia de las estacas estuvo arriba del $80 \%$, con los mayores porcentajes para la del Nevado de Toluca. De manera similar para las estacas enraizadas que alcanzaron porcentajes por arriba del $65 \%$; y también para la longitud de raíz que siempre mantuvieron un tamaño mayor a los 8 cm (cuadro 4).

En la producción de raíces de primer y segundo, las estacas del Cofre de Perote incrementaron la producción de las de primer orden en más de $30 \%$, pero disminuyeron su producción en las de segundo orden en más del $25 \%$, con respecto a las otras dos poblaciones (cuadro 4).

Interacción entre AIB y población. Solo en la producción de raíces secundarias se presentó interacción entre los dos factores. Con la aplicación de AIB se incrementó la formación de raíces secundarias con respecto al testigo en todas las dosis y poblaciones; aunque no en la misma proporción. Sin embargo, la máxima dosis de AIB (10.000ppm) redujo el promedio de este tipo de raíces en dos de las poblaciones, Monte Tláloc y Nevado de Toluca, en relación a la dosis media (5.000 ppm); mientras que en la población de Cofre de Perote la producción de estas raíces se incrementó (figura 2).

Trasplante de estacas enraizadas. A pesar de las diferencias señaladas en la morfología de la raíz de las estacas a causa del AIB y la población; después de 3 meses del trasplante a contenedor forestal el porcentaje de supervivencia fue del $100 \%$ en todos los tratamientos de AIB y poblaciones. Tres meses más tarde se registró solo un $3 \%$ de mortalidad global, sin relación con los factores en estudio, población y dosis de AIB.

\section{DISCUSIÓN}

Efecto de la concentración de auxina. El decremento en la supervivencia en las estacas de $P$. hartwegii indicó cierto grado de toxicidad del AIB en la concentración más alta (10.000 ppm); ya que en otras especies como Pinus patula, las concentraciones mayores de $8.000 \mathrm{ppm}$ de esta auxina no afectaron la supervivencia de las estacas y respondieron favorablemente con la formación de raíces (Rivera-Rodríguez et al. 2016). Aunque el AIB es la auxina sintética ideal para trabajos de enraizamiento, por su baja movilidad, estabilidad y sobre todo baja toxicidad (Ragonezi et al. 2010); también puede resultar tóxica en concentraciones altas para algunas especies y tipo de propágalo (De Bona et al. 2012). De acuerdo con nuestros resultados, para evitar daño y muerte de las estacas de $P$. hartweggi no se recomienda el uso de concentraciones de AIB de 10.000 ppm.

Sin embargo, la capacidad promotora del AIB en la inducción de raíces adventicias en las estacas fue evidente con el tratamiento de 5.000 ppm de AIB. Esta concen-

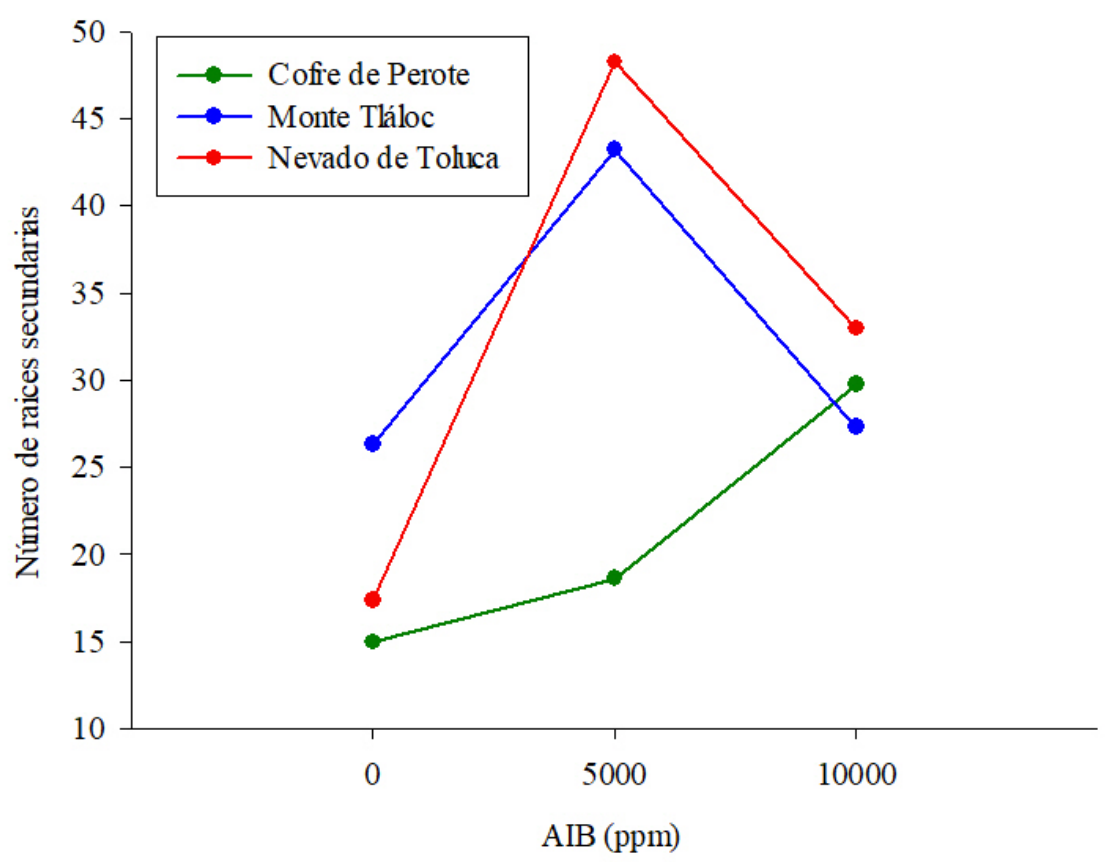

Figura 2. Medias del número de raíces secundarias de las estacas enraizadas Pinus hartwegii para cada subgrupo resultante de combinar cada nivel del factor concentración de ácido Indolbutirico (AIB: 0, 5.000 y 10.000 ppm) aplicado con cada nivel del factor población (Cofré de Perote, Veracruz, Monte Tláloc, Edo. de México y Nevado de Toluca; Edo. de México).

Interaction between populations and auxin levels for number of adventitious roots in Pinus hartwegii. CP: Cofré de Perote, Veracruz; MT: Monte Tlaloc, Edo. from Mexico; NT: Nevado de Toluca; Edo. from Mexico; AIB: Indole Butyric Acid. 
tración resultó ideal para la especie ya que no se afectó la supervivencia y se logró amplificar la repuesta de las estacas durante la morfogénesis de las raíces adventicias. $\mathrm{Al}$ margen de la respuesta al AIB, se debe señalar que las estacas de $P$. hartwegii mostraron tener buena capacidad para enraizar sin el uso de auxinas exógenas. Es probable que tanto la auxina endógena como la condición del tejido de la estaca hayan contribuido en su respuesta al enraizamiento. Díaz-Sala (2021) señala que, en las especies de pino, la acumulación de auxina en los tejidos de la estaca es una respuesta natural cuando los tejidos son competentes. También menciona, que cuando el tejido pierde esta característica de competencia, el flujo polar de auxinas endógenas hacia el sitio de enraizamiento puede ser inhibido y afectar el proceso de inducción de raíces. Por lo que la aplicación de auxinas exógenas es necesaria para mejorar la respuesta al enraizamiento de las estacas de las especies de pino, ya que en ausencia de auxinas exógenas el porcentaje de enraizamiento es bajo (Cuevas-Cruz et al. 2015, Rivera et al. 2016, Riov et al. 2020).

Además, en este trabajo también se confirmó que el AIB mejoró la producción de raíces primarias y secundarias; efecto que ha sido consistente en las estacas de varias especies forestales maderables, tanto en coníferas como en latifoliadas (Bielenin 2003). En investigaciones con especies de Pinus y Eucalyptus, el AIB estimuló la producción de mayor número de raíces y de otros atributos relacionados con la morfología de la raíz (Navarrete-Luna y VargasHernández 2005, Majada et al. 2011). Se ha mencionado que el AIB incrementa y mejora el transporte basipétalo de asimilados, hacía sitios de alta demanda, promoviendo el incremento en el número de raíces (Salmi y Hesami 2016). La producción de raíces de primer y segundo orden en las estacas es muy importante ya que le proporciona mayor superficie de captación de agua y translocación de nutrientes, lo que evita que mueran y mejora el crecimiento y desarrollo de la planta (Davis y Jacobs 2005). Por tanto, entre menos tiempo tarden en formarse las raíces, la probabilidad para que las estacas sobrevivan se incrementa y se concluya el proceso para conformar una planta completa.

Aunque en algunos trabajos sobre enraizamiento de estacas se ha señalado que el AIB si influye en el crecimiento en longitud de la raíz principal (Santelices y García 2003), en el presente trabajo no hubo diferencias para esta variable en ninguno de los tratamientos de AIB aplicados. Sin embargo, las estacas que lograron enraizar presentaron una raíz bien formada y con tamaño aceptable $(7,1-9,4 \mathrm{~cm})$, para desarrollar las funciones que le permitan a la planta sobrevivir durante la fase de vivero y el establecimiento inicial en condiciones de campo. Es común que las estacas desarrollen más de tres raíces adventicias, de las cuales ninguna adquiere el papel de raíz principal (Cuevas-Cruz et al. 2015). Ventajas o desventajas de este aspecto deben ser evaluadas tanto después del trasplante de la estaca enraizada a contenedor como en la plantación en campo a mediano y largo plazo.
Efecto de la población. La falta de variación entre las poblaciones, respecto a los porcentajes de supervivencia y de estacas enraizadas, probablemente se debe a la calidad de las estacas producidas en las plantas donadoras. Todas las plantas fueron manejadas con el mismo régimen de riegos y fertilización, por lo que la calidad de la estaca estaría determinando el éxito en el enraizamiento y en consecuencia de su supervivencia. Por otro lado, P. hartwegii presenta un marcado estado cespitoso durante su ontogenia temprana, lo cual se ha asociado con una adaptación a los incendios. Se ha documentado ampliamente que las especies de pino que presentan este estado, acumulan gran cantidad de sustancias de reserva, particularmente en la zona del cuello de raíz (Paula et al. 2016). Durante el proceso de enraizamiento, la supervivencia de la estaca depende de los factores ambientales y de las sustancias de reserva, como los carbohidratos (Husen 2012). Para este estudio, se utilizaron estacas basales cercanas al cuello de raíz fueron usadas, por lo que es muy probable que tanto el manejo y el origen de las estacas en la planta donadora hayan influido en la concentración de carbohidratos contenidos en los tejidos de las estacas, beneficiando su supervivencia y enraizamiento (Otiende et al. 2017), independientemente del efecto de la población. Sin embargo, es necesario hacer más investigación sobre la presencia de carbohidratos en las estacas y su relación con el desarrollo las raíces adventicias en esta especie.

Por otro lado, la variación observada en la formación de raíces primarias y secundarias entre poblaciones podría ser una compensación de la planta, ya que con menor número de raíces primarias se desarrollan más raíces secundarias y viceversa (Nascimento et al. 2019). Las estacas de la población del Cofre de Perote tuvieron mejor respuesta en la producción de raíces primarias; mientras que las del Nevado de Toluca y Monte Tláloc lo fueron para las raíces secundarias. Algunos estudios han señalado que la producción de raíces en las estacas es muy variable y depende de la calidad de la estaca y del genotipo, inclusive la variación es común entre estacas provenientes de la misma población (Aparicio-Rentería et al. 2014). Esta respuesta se ha mencionado en otras coníferas, incluso entre clones; donde se argumenta que se debe a los diferentes niveles de recalcitrancia al enraizamiento existentes entre genotipos (De Oliveira et al. 2020).

Interacción entre AIB y poblaciones. La interacción detectada entre factores para la producción de raíces secundarias demuestra la variación en sensibilidad al AIB, cuando la dosis se incrementa de 5.000 a 10.000 ppm entre poblaciones. Algunos autores han indicado que el uso de altas concentraciones de AIB induce la síntesis de etileno, el cual puede inhibir el desarrollo de raíces adventicias; dependiendo de la especie, genotipo y sensibilidad del tejido (Veloccia et al. 2016). Esto puede explicar en parte la causa del decremento observado en las raíces secundarias de las estacas del Monte Tláloc y Nevado de Toluca, con respecto al efecto contrario de las del Cofre de Perote. 
Trasplante de estacas enraizadas. Las diferencias encontradas en la morfología de la raíz de las estacas no influyeron en la supervivencia de la plántula durante los primeros seis meses de su trasplante. Lo cual indica la buena calidad y funcionalidad de las raíces adventicias formadas en las estacas. La morfología de raíces tiene gran relevancia en los procesos de aclimatación que definen el establecimiento y supervivencia de las plantas en condiciones de campo (Pernot et al. 2019). Por lo que, es necesario continuar la evaluación del desarrollo y crecimiento de las plantas enraizadas en condiciones de campo, para complementar la información y proponer el uso de esta técnica en la producción de plantas de $P$. hartwegii.

\section{CONCLUSIONES}

Las estacas de $P$. hartwegii muestran una alta capacidad de generar raíces adventicias, capacidad que aumenta con la aplicación de AIB. En las estacas de las tres poblaciones estudiadas se recomienda utilizar la dosis de $5.000 \mathrm{ppm}$ para optimizar el enraizamiento y producción de planta. Evitar dosis mayores de AIB ya que tienden a generar toxicidad y afectar la supervivencia de las estacas; a pesar de que, en estacas enraizadas, según su origen (Cofre de Perote), pueden incrementar la producción del número de raíces secundarias. La propagación a través del enraizado de estacas es una alternativa viable para resolver la escaza producción de semilla y generar planta a gran escala de las tres poblaciones estudiadas de $P$. hartwegii, requeridas por los programas nacionales de reforestación. Sin embrago, la evaluación en campo de la planta producida vegetativamente será necesaria para determinar la factibilidad de la técnica y validar la producción de planta de $P$. hartwegii de manera masiva.

\section{AGRADECIMIENTOS}

El autor del trabajo Agradece al Consejo Nacional de Ciencia y Tecnología (CONACYT) por la beca otorgada para realizar sus estudios de Maestría en Ciencias. Al Colegio de Postgraduados por el financiamiento para la realización del presente trabajo a través del presupuesto AAA y proyecto aprobado en la convocatoria 2018 financiado por el Fideicomiso a las LGAC; y a todos los ejidos incluidos en la montaña del Tlácolc del municipio de Texcoco; así como a los del Nevado de Toluca y Veracruz por las facilidades brindadas para la recolecta de semillas usadas en la presente investigación.

\section{REFERENCIAS}

Alfaro-Ramírez FU, JE Ramírez-Albores, JJ Vargas-Hernández, S Franco-Maass, M Pérez Suárez. 2020. Potential reduction of Hartweg's Pine (Pinus hartwegii Lindl.) geographic distribution. PLoS ONE 15(2): e0229178. . DOI: https://doi. org/10.1371/journal.pone.0229178.

Aparicio-Rentería A, SF Juárez-Cerrillo, LR Sánchez-Velásquez. 2014. Propagación por enraizamiento de estacas y conserva- ción de árboles plus extintos de Pinus patula procedentes del norte de Veracruz, México. Madera y Bosques 20(1): 85-96.

Bautista-Ojeda GI. 2020. Efecto del método y tiempo de aplicación de ácido indol-3-butírico (AIB) en el enraizado de estacas de Pinus patula. Tesis de Maestría en Ciencias Forestales. Texcoco, Estado de México, México. Posgrado en Ciencias Forestales, Colegio de Postgraduados. 56 p.

Bielenin M. 2003. Rooting and gas exchange of conifer cuttings treated with indolbutyric acid. Journal of Fruit and Ornamental Plant Research 11: 99-105.

Bonga JM, KK Klimaszewska, P Von Aderkas. 2010. Recalcitrance in clonal propagation, in particular of conifers. Plant Cell Tissue and Organ Culture 100: 241-254. DOI: https:// doi.org/10.1007/s11240-009-9647-2.

Cuevas-Cruz JC, M Jiménez-Casas, J Jasso-Mata, P Pérez-Rodríguez, J López-Uptón, A Villegas-Monter. 2015. Asexual propagation of Pinus leiophylla Schiede ex Schltdl. et Cham. Revista Chapingo Serie Ciencias Forestales y del Ambiente 21(1): 81-95. DOI: https://doi.org/10.5154/r. rchscfa.2014.08.033.

Davis A, D Jacobs. 2005. Quantifying root system quality of nursery seedlings and relationship to outplanting performance. New Forests 30: 295-311. DOI: https://doi.org/10.1007/ s11056-005-7480-y.

De Bona CM, MA Masetto, C Deschamps, LA Biasi. 2012. Rooting induction of different Lavandula angustifolia accessions by auxin application. Semina Ciências Agrárias 33(1): 75-182. DOI: https://doi.org/10.5433/1679$0359.2012 \mathrm{v} 33 \mathrm{n} 1 \mathrm{p} 175$.

De Oliveira PM, AÂ Camargo, MC Navroski, LM De Oliveira, D Felippe, C Moraes. 2020. Mini-cuttings rootings of Sequoia sempervirens at different IBA concentrations and clones. Floresta 50 (2): 1279 - 1286. DOI: https://doi.org/10.5380/ rf.v50i2.62579.

Díaz-Sala C. 2021. A perspective on adventitious root formation in tree species. Plants 10 (3): 486-494. DOI: https://doi. org/10.3390/plants10030486.

Escamilla-Hernández N, Aldrete A, Vargas-Hernández JJ, Villegas-Monter A, López-López MA. 2020. Vegetative propagation of Pinus patula SCHIEDE ex SCHLTDL. et CHAM. in different substrates. Revista Fitotecnia Mexicana 43 (2): 215-221. DOI: https://doi.org/10.35196/rfm.2020.2.215.

Flores-García A, ME Romero-Sánchez, R Pérez-Miranda, F Moreno-Sánchez. 2020. Potencial de restauración de bosques de coníferas en zonas de movimiento de germoplasma en México. Revista Mexicana de Ciencias Forestales 12 (63): 4-27. DOI: https://doi.org/10.29298/rmcf.v12i63.813.

Gutiérrez E, I Trejo. 2014. Efecto del cambio climático en la distribución potencial de cinco especies arbóreas de bosque templado en México. Revista Mexicana de Biodiversidad 85: 179 -188. DOI: https://doi.org/10.7550/rmb.37737.

Hakamata T, Y Hiraoka, S Yamamoto, K Kato. 2016. Effect of family, crown position, number of winter buds, fresh weight and the length of needle on rooting ability of Pinus thunbergii Parl. cuttings. iForest 9: 370 - 374. DOI: https://doi. org/10.3832/ifor1661-008.

Husen A. 2012. Changes of soluble sugars and enzymatic activities during adventitious rooting in cuttings of Grewia optiva as affected by age of donor plants and auxin treatments. American Journal of Plant Physiology 7: 1-16. DOI: https://doi.org/10.3923/ajpp.2012.1.16. 
Majada J, C Martínez-Alonso, I Feito, A Kidelman, I Aranda, R Alia. 2011. Mini-cuttings: an effective technique for the propagation of Pinus pinaster Ait. New Forests 41: 399 412. DOI: https://doi.org/10.1007/s11056-010-9232-x.

Matsunaga K, Ohira M. 2019. Effect of cutting size on rooting ability and first year growth of Pinus thunbergii in nursery containers. Journal of Forest Research 24 (6): 356-364, DOI: https://doi.org/10.1080/13416979.2019.1675252.

Navarrete-Luna M, JJ Vargas-Hernández. 2005. Propagación asexual de clones de Eucalyptus camaldulensis Dehnh. utilizando radix en diferentes concentraciones. Revista Chapingo Serie Ciencias Forestales y del Ambiente 11(2): 111 - 116.

Nascimento DC, M Dini, NV Sampaio, MW Schuch. 2019. In vitro multiplication and rooting of grapevine: Culture media and plant growth regulators. Plant Cell Culture Micropropagation 15(1): 1-7. DOI: https://doi.org/10.46526/ pccm.2019.v15i1.136.

Nugrahanto G, M Naiem, S Indrioko, E Faridah, W Widiyanto. 2019. Rooting ability of Pinus merkusii with high resin yield from shoot cutting. Jurnal Pemuliaan Tanaman Hutan 13(2): 71-83. DOI: https://doi.org/10.20886/ jpth.2019.13.2.71-83.

Otiende MA, Nyabundi JO, Ngamau K, Opala P. 2017. Effects of cutting position of rose rootstock cultivars on rooting and its relationship with mineral nutrient content and endogenous carbohydrates. Scientia Horticulturae 225: 204-212. DOI: https://doi.org/10.1016/j.scienta.2017.07.009.

Pacurar DI, I Perrone, C Bellini. 2014. Auxin is a central player in the hormone cross-talks that control adventitious rooting. Physiologia Plantarum 151: 83 - 96. DOI: https://doi. org/10.1111/ppl.12171.

Paula S, PI Naulin, C Arce, C Galaz, JG Pausas. 2016. Lignotubers in Mediterranean basin plants. Plant Ecology 217: 661 - 676. DOI: https://doi.org/10.1007/s11258-015-0538-9.

Pernot C, N Thiffault, A DesRochers. 2019. Influence of Root System Characteristics on Black Spruce Seedling Responses to Limiting Conditions. Plants 8 (70): 1-17. DOI: https://doi.org/10.3390/plants8030070.
Ragonezi C, K Klimaszewska, MR Castro, M Lima, P De Oliveira, MA Zavattieri. 2010. Adventitious rooting of conifers: influence of physical and chemical factors. Trees 24: 975 - 992. DOI: https://doi.org/10.1007/s00468-0100488-8

Riov J, H Fox, R Attias, G Shklar, L Farkash-Haimb, R Sitbon, Y Moshe, M Abu-Abied, E Sadot, E David-Schwartz. 2020. Improved method for vegetative propagation of mature Pinus halepensis and its hybrids by cuttings. Israel Journal of Plant Sciences 67(1-2):1:15. DOI: https://doi. org/10.1163/22238980-20191118.

Rivera-Rodríguez MO, JJ Vargas-Hernández, J López-Upton, A Villegas-Monter, M Jiménez-Casas. 2016. Enraizamiento de estacas de Pinus patula. Revista Fitotecnia Mexicana 39(4): 385-392.

Salmi MS, M Hesami. 2016. Time of collection, cuttingages, auxin types and concentrations influence rooting Ficus religiosa L. stem cuttings. Journal Applied Environmental Biological Sciences 6: 124 - 132.

Santelices R, C Garcia. 2003. Efecto del ácido indolbutírico y la ubicación de la estaca en el rebrote de tocón sobre la rizogénesis de Nothofagus alessandrii Espinosa. Bosque 24(2): 53-61. DOI: https://doi.org/10.4067/s071792002003000200006.

Tejeda-Landero VM, LR Sánchez-Velásquez, H Viveros-Viveros, A Aparicio-Rentería, R Flores-Peredo. 2019. Seed bank formation and removal of Pinus hartwegii (Pinaceae) seeds along an altitudinal gradient in the Cofre de Perote National Park, Veracruz, Mexico. Botanical Sciences 97(4): 623-629. DOI: https://doi.org/10.17129/botsci. 2204.

Veloccia A, L Fattorini, RL Della, A Sofo, S D'Angeli, C Betti, G Falasca, MM Altamura. 2016. Ethylene and auxin interaction in the control of adventitious rooting in Arabidopsis thaliana. Journal of Experimental Botany 67(22): 6445-6458. DOI: https://doi.org/10.1093/jxb/erw415.

White T, T Adams, Neale D B. 2007. Forest Genetics. CABI Publishing, Cambridge, MA, USA. 662 p.

Recibido: 30/11/20

Aceptado: 08/09/21 
